\title{
A METHODOLOGY FOR CREATING INTELLIGENT WHEELCHAIR USERS' PROFILES
}

\author{
Brígida Mónica Faria ${ }^{1,2,5}$, Sérgio Vasconcelos ${ }^{3,5}$, Luís Paulo Reis ${ }^{4,5}$ and Nuno Lau ${ }^{2}$ \\ ${ }^{I}$ Escola Superior de Tecnologia da Saúde do Porto, Instituto Politécnico do Porto, Vila Nova de Gaia, Portugal \\ ${ }^{2}$ Dep. Elect., Telecomunicações e Informática (DETI/UA), \\ Inst. Eng. Electrónica e Telemática de Aveiro, Universidade de Aveiro, Aveiro, Portugal \\ ${ }^{3}$ Dep. Eng. Informática, Faculdade de Engenharia, Universidade do Porto, Porto, Portugal \\ ${ }^{4}$ Dep. Sistemas de Informação, Escola de Engenharia, Universidade do Minho, Guimarães, Portugal \\ ${ }^{5}$ Laboratório de Inteligência Artificial e Ciência de Computadores, Universidade do Porto, Porto, Portugal
}

Keywords: Intelligent wheelchair, Users' profile, Adaptive interface.

Abstract: Intelligent Wheelchair (IW) is a new concept aiming to allow higher autonomy to people with lower mobility such as disabled or elderly individuals. Some of the more recent IWs have a multimodal interface, enabling multiple command modes such as joystick, voice commands, head movements, or even facial expressions. In these IW it may be very useful to provide the user with the best way of driving it through an adaptive interface. This paper describes the foundations for creating a simple methodology for extracting user profiles, which can be used to adequately select the best IW command mode for each user. The methodology is based on an interactive wizard composed by a flexible set of simple tasks presented to the user, and a method for extracting and analyzing the user's execution of those tasks. The results achieved showed that it is possible to extract simple user profiles, using the proposed method. Thus, the approach may be further used to extract more complete user profiles, just by extending the set of tasks used, enabling the adaptation of the IW interface to each user's characteristics.

\section{INTRODUCTION}

The fraction of population with physical disabilities has earned more relevance and has attracted the attention of international health care organizations, universities and companies interested in developing and adapting new products. The actual tendency reflects the demand for an increase on health and rehabilitation services, in a way that senior and handicapped individuals might become more and more independent performing quotidian tasks.

Regardless the age, mobility is a fundamental characteristic for every human being. Children with disabilities are very often deprived of important opportunities and face serious disadvantages compared to other children. Adults who lose their independent means of locomotion become less self sufficient, raising a negative attitude towards them. The loss of mobility originates obstacles that reduce the personal and vocational objectives (Simpson, 2005). Therefore it is necessary to develop technologies that can aid this population group, in a way to assure the comfort and independence of the elderly and handicapped people. Wheelchairs are important locomotion devices for those individuals. There is a growing demand for safer and more comfortable wheelchairs, and therefore, a new Intelligent Wheelchair (IW) concept was introduced. However, most of the Intelligent Wheelchairs developed by distinct research laboratories (Simpson, 2005), have hardware and software architectures very specific for the used wheelchair model/developed project and are typically very difficult to configure in order for the user to start using them.

The rest of the paper is organized as follows. Section 2 presents the state of art on intelligent wheelchairs. Section 3 contains a description of the users' interfaces already developed and how the interface is integrated in our work. Section 4 presents the implementation and methodology for creating an intelligent wheelchair user interface. The experiments and the results achieved are presented in section 5. Finally some conclusions and future work is described in the last section. 


\section{INTELLIGENT WHEELCHAIRS}

In the last years several prototypes of Intelligent Wheelchairs (Figure 1) have been developed and many scientific work has been published (Braga et al., 2009) (Reis et al., 2010) in this area. Simpson (Simpson, 2005) provides a comprehensive review of IW projects with several descriptions of intelligent wheelchairs. The main characteristics of an IW are (Braga et al., 2009) (Jia, 2007): interaction with the user using distinct types of devices such as joysticks, voice interaction, vision and other sensor based controls like pressure sensors; autonomous navigation with safety, flexibility and obstacle avoidance capabilities and communication with others devices such automatic doors and other wheelchairs.

The first project of an autonomous wheelchair for physical handicapped was proposed by Madarasz in 1986 (Madarasz, 1986). It was planned as a wheelchair with a micro computer, a digital camera and an ultra-sound scanner with the objective of developing a vehicle that could move around in populated environments without human intervention. Hoyer and Holper (Hoyer and Holper, 1993) presented a modular control architecture for an Omni-directional wheelchair. The characteristics of NavChair (1996), such as the capacity of following walls and avoid obstacles by deviation are described in (Simpson, 1998) (Bell et al., 1994) (Levine, 1999). Miller and Slak (Miller and Slak, 1995) (Miller, 1998) proposed the system Tin Man I with three operation modes: one individual driving a wheelchair with automatic obstacles deviation; moving through-out a track and moving to a point $(\mathrm{x}, \mathrm{y})$. This kind of chair evolved to Tin Man II which included advanced characteristics such as storing travel information, return to the starting point, follow walls, pass through doors and recharge battery. Wellman (Wellman et al., 1994) proposed a hybrid wheelchair equipped with two extra legs in addition to its four wheels, to allow stair climbing and movement on rough terrain. FRIEND is a robot for rehabilitation which consists of a motorized wheelchair and a MANUS manipulator (Borgerding et al., 1999) (Volosyak et al., 2005). In this case, both the vehicle and the manipulator are controlled by voice commands. Some projects present solutions for quadriplegic individuals, where facial expressions recognition is used to control the wheelchair (Jia et al., 2007) ( $\mathrm{Ng}$ and Silva, 2001) (Adachi et al., 1998). In 2002, Pruski presented
VAHM, a user adapted intelligent wheelchair (Pruski et al., 2002).

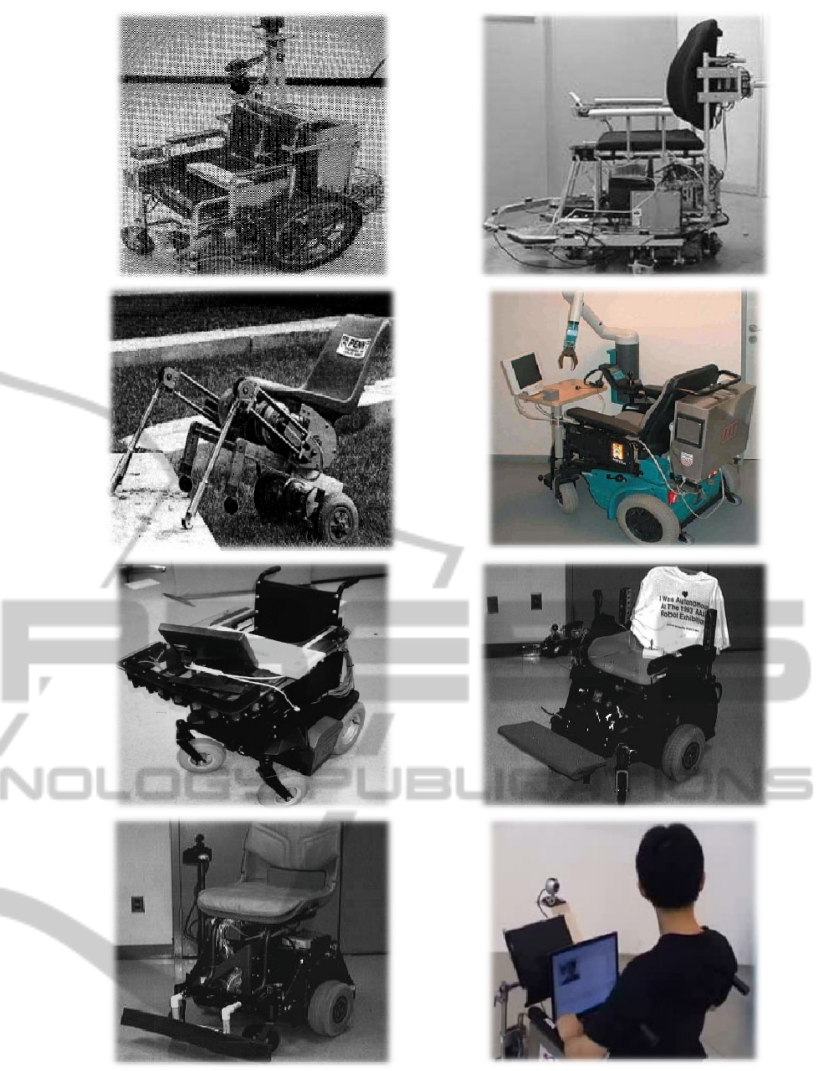

Figure 1: Several prototypes of Intelligent Wheelchairs.

Satoh and Sakaue (Satoh and Sakaue, 2007) presented an omni-directional stereo vision-based IW which detects both the potential hazards in a moving environment and the postures and gestures of a user, using a stereo omni-directional system, which is capable of acquiring omni-directional color image sequences and range data simultaneously in real time. In 2008 John Spletzer studied the performance of LIDAR based localization for docking an IW system (Spletzer et al., 2008) and in 2009 Horn and Kreutner (Horn and Kreutner, 2009) showed how the odometric, ultrasound, and vision sensors are used in a complementary way in order to locate the wheelchair in a known environment. In fact, the research on IW has suffered a lot of developments in the last few years. Some IW prototypes are controlled with "thought". This type of technology uses sensors that pick up electromagnetic waves of the brain (Hamagami and Hirata, 2004) (Lakany, 2005) (Rebsamen, 2006). 


\subsection{IntellWheels Project}

This section presents a brief overview of the Intelligent Wheelchair project that is being developed at the Faculty of Engineering of the University of Porto (FEUP) in collaboration with INESC-P and the University of Aveiro. Also, the primary results of this project that have already been published are also presented. The main objective of the IntellWheels Project is to develop an intelligent wheelchair platform that may be easily adapted to any commercial wheelchair and aid any person with special mobility needs. Initially, an evaluation of distinct motorized commercial wheelchair platforms was carried out and a first prototype was developed in order to test the concept. The first prototype was focused on the development of the modules that provide the interface with the motorized wheelchair electronics using a portable computer and other sensors.
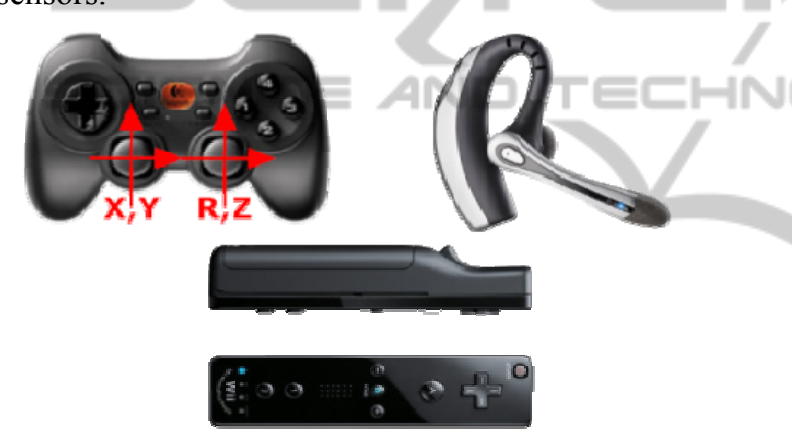

Figure 2: Generic gamepad, headset with microphone and Nintendo Wii Remote.

Several different modules have been developed in order to allow different ways of conveying commands to the IW. These include, for example, joystick control with USB, voice commands, control with head movements and gestures, and facial expressions recognition (Faria et al., 2007). Figure 2 shows the three commands already available in the IntellWheels IW. The project research team considered the difficulty that some patients have while controlling a wheelchair using traditional commands such as the traditional joystick. Therefore, new ways of interaction between the wheelchair and the user have been integrated, creating a system of multiple entries based on a multimodal interface. The system allows users to choose which type of command best fits their needs, increasing the level of comfort and safety.

Another possibility enabled by a system of multiple entries is the use of software for intelligent control of inputs. This application has the task of determining the confidence level of each of the entries, or even cancels them if it detects the presence of conflicts or noise in the surrounding environment. For example, in a very dark or very bright room, where the patient's face is not fully recognized, the intelligent control of inputs would decrease the degree of confidence of this type of software commands sent by the recognition of facial expressions, and would provide greater importance to the joystick, voice and/or head movements based commands.

\section{USER PROFILES}

In addition to the wide variety of disabilities that cause different types of limited mobility, each person has specific characteristics, which may be related to physical and cognitive factors. Thus, individuals with similar symptoms may have significant differences. It is fair to say that characteristics such as moving the head, ability to pronounce words, ability to move the hand and fingers, can vary substantially from individual to individual. Similarly, the time of learning and proficiency in using assistive devices may also vary greatly.

\subsection{User Interfaces}

The interface between a human and a computer is called a user interface and it is a very important part of any computerized system. Moreover, an adaptive user interface (Langley, 1999) is a software entity that improves its ability to interact with a user by constructing a user model based on past experience with that user. The emerging area of adaptive and intelligent user interfaces has been exploring applications in which these paradigms are useful and facilitate the human machine communication (Ross, 2000). In fact, if an intelligent user interface has a model of the user, this user model can be used to automatically adapt the interface. Additionally, adaptive user interfaces may use machine learning techniques to improve the interaction with individuals in order to have the users reach their goal more easily, faster and with a higher level of satisfaction. It is also essential for an adaptive interface to obtain knowledge included in four distinct domains: knowledge of the user; knowledge of the interaction (modalities of interaction and dialogue management); knowledge of the task/domain; and knowledge of the system characteristics (Norcio and Stanley, 1989). 


\subsection{Adaptive Interfaces}

Ross (Ross, 2000) presented a comprehensive classification of adaptive/intelligent interfaces. His classification contains three main classes. The first class involves the addition of adaptation to an existing direct manipulation interface. Examples of this class are adding extra interface objects in order to hold the predicted future commands or designing an interface with multiple commands. The second class is composed by interfaces acting as an intermediary between the user and the direct manipulation interface by filtering information or generating suggested data values. The third class is composed by the agent interfaces, in which autonomous agents (Maes, 1996) (Wooldridge, 2002) can provide pro-active support to the user, typically can make suggestions and give advice.

It is also mentioned that many intelligent interfaces can be viewed as adaptive user interfaces, because they change their behaviour to adapt to an individual or assignment (Ross, 2000). Another taxonomy defended by Langley (Langley, 1997) for adaptive user interfaces (AUI) is based on separating them into two groups: Informative Interfaces and Generative Interfaces. The first class selects information for the user and presents the items he will find interesting or practical. The second process tries to generate a useful knowledge structure like spread-sheets, document preparation or drawing packages.

Also, in the literature, another class of adaptive interfaces is presented and studied. This class is designated as Programming by Demonstration (Cypher and Halbert, 1994) (Ross, 2000). This class is distinct from the previous since generative interfaces produce data values but programming by demonstration systems produce commands with arguments.

\subsection{Intellwheels User Profile}

Tracing a user diagnostic can be very useful to adjust certain settings allowing for an optimized configuration and improved interaction between the user and the multimodal interface.

Accordingly, the Intellwheels Multimodal Interface should contain a module capable of performing series of training sessions, composed of small tests for each input modality. These tests may consist, for example, of asking the user to press a certain sequence of buttons on the gamepad, or to move one of the gamepads' joysticks to a certain position. Another test may consist in asking the user to pronounce a set of voice commands, or to perform a specific head movement. Figure 3 shows where the user needs to click to start the User Profiler module.

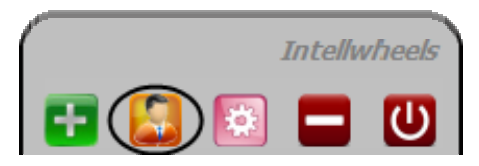

Figure 3: Starting user's profile module.

The tests should be performed sequentially and should have an increasing difficulty. Additionally, the tests should be reconfigurable and extensible. Finally, the tests sets and theirs results should be saved on a database, accessible by the Intellwheels Multimodal Interface. Therefore, the following user characteristics should be extracted. These characteristics are separated in two different types: quantitative and qualitative. The quantitative measures consist of: the time taken to perform a full button sequence; the average time between pressing two buttons; the average time to place a gamepad analogical switch on a certain position; the average time to position the head on a certain position; the trust level of speech recognition; maximum amplitude achieved with the gamepad analogical switches in different directions; maximum amplitude achieved with the head in different directions and number of errors made using the gamepad. Using the quantitative measures, the following qualitative measures should be estimated: user ability to use the gamepad buttons; user ability to perform head movements and user ability to pronounce voice commands.

At the end of the training session, the tracked user information should be saved to an external database, containing the users' profile. The user profile can be used to improve security, by defining, for each user, a global trust level for each input modality. The trust level can be used to advice the user of each modality to use, at the creation of a new association. Also, it could be useful to activate confirmation events whenever a user requests a certain output action using an input level with a low trust level.

\section{IMPLEMENTATION}

This section presents the implementation for the proposed User Profile feature. Firstly, it explains the approach followed to specify which the test sets are going to be loaded by the module responsible for tracking the users' profile. Secondly, we show the simple profiling methods that were implemented to 
create in future a user classification. Following, it is explained how the extracted information was used to adjust certain settings of the interface. Finally, a demonstration of how the profile is stored to enable future use is also made.

\subsection{Definition of the Sets}

To perform the measures described in the previous section, a simple XML grammar was defined. It implements four configurable distinct test types: sequences of gamepad buttons; voice commands; positions for both joysticks and positions for head.

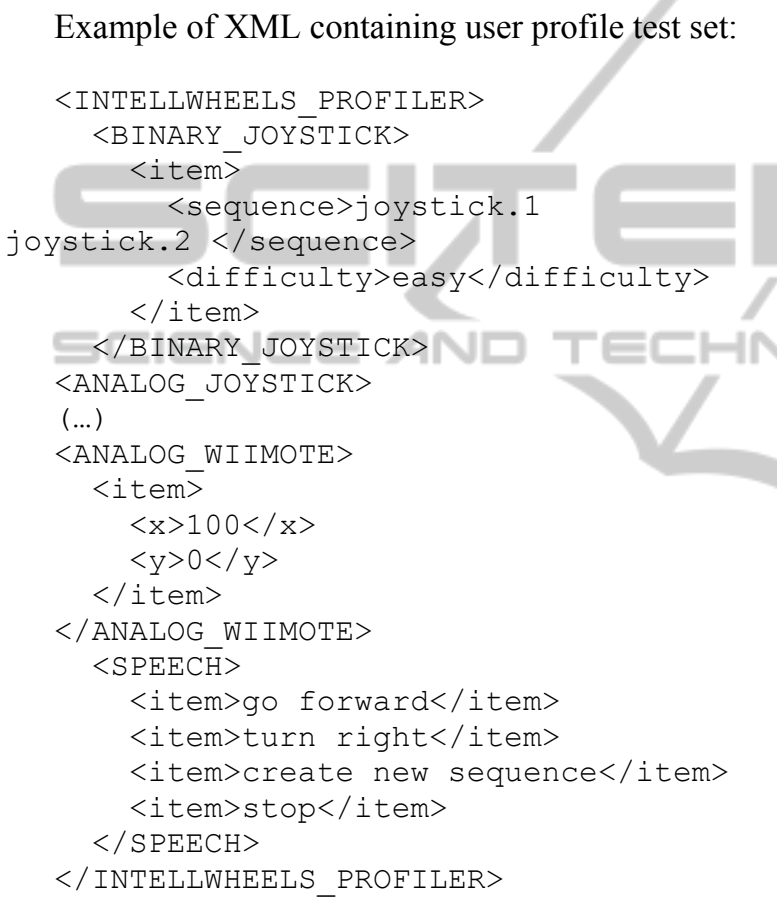

The proposed XML grammar makes it possible for an external operator to configure a test set that they may find appropriate for a specific context or user. When a user starts the training session, the four different types of tests are iterated. In order to attain a consistent classification of the user, the defined grammar should be sufficiently extensive. The test set present on the XML file is iteratively shown to the user. It starts by asking the user to perform the gamepad button sequence as can be observed in Figure 4.

When the user ends the first component of the user profiler module, the navigation assistant asks the user to pronounce the voice commands stored in the XML. Also, the quantitative results for the gamepad buttons test are presented.

The last part of the user profiler test is shown in

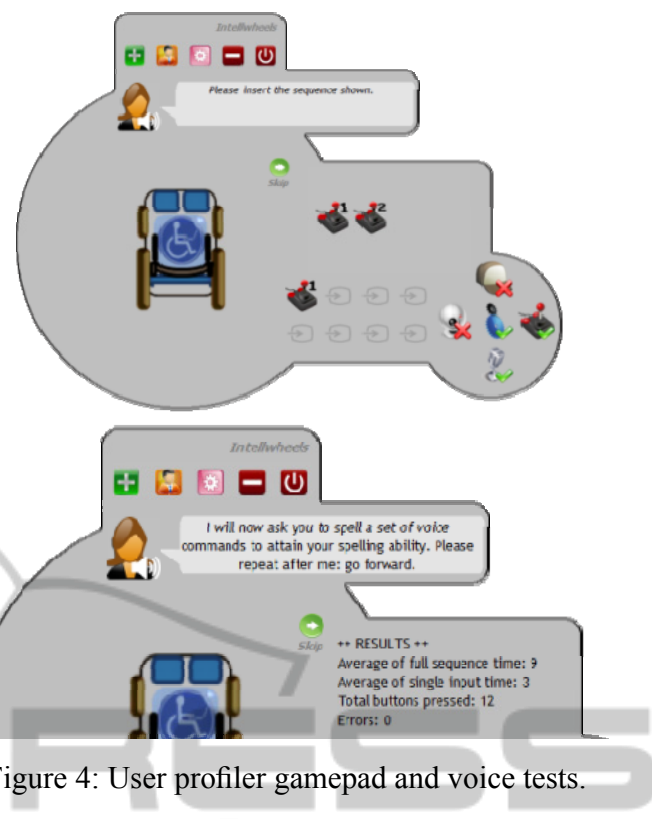

Figure 5. The user is invited to place the gamepad's joystick into certain positions. A similar approach is used for the head movements test.

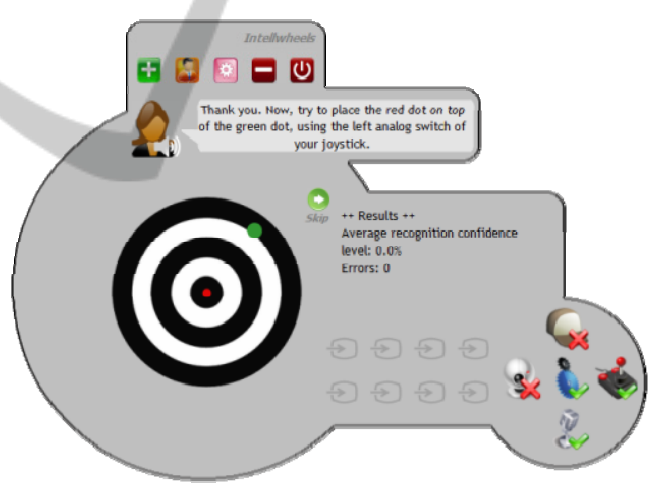

Figure 5: User profiler joystick test.

To define the user proficiency in using the gamepad buttons, a very simple method was implemented. Each sequence defined on the grammar should have an associated difficulty level (easy, medium or hard). The difficulty type of a sequence may be related to its size, and to the physical distance between the buttons on the gamepad. Since the layout of a generic gamepad may change depending on the model, defining whether or not a sequence is of easy, medium or difficulty level is left to the operator.

When the user completes the gamepad sequences training part, an error rate is calculated for each of the difficulty levels. If these rates are higher than a minimum acceptable configurable value, the user classification in this item is immediately defined. 
This classification is then used to turn on the security feature, which is characterized by a confirmation event performed by the navigation assistant. For a grammar with 5 sequences of difficulty type easy, the maximum number of accepted errors would be 1 . If the user fails more than one sequence, the confirmation event is triggered for any input sequence, of any difficulty type, and the gamepad training session is terminated. If the error rate for the easy type is less than $20 \%$ $(=1 / 5)$ the training with the sub-set composed by the sequences of medium difficulty is initiated. At the end, a similar method is applied. If the error rate for the medium level is higher than $30 \%$, the confirmation is triggered for the medium and hard levels of difficulty, and the training session is terminated. Finally, if the user makes it to the last level of difficulty, the training for the hard sequences sub-set is started. If the error rate is higher than $50 \%$, the confirmation event is triggered only for sequences with a hard difficulty level. The best scenario takes place when the user is able to surpass the maximum accepted error rates for all the difficulty levels. In this situation, the confirmation event is turned off, and an output request is immediately triggered for any kind of input sequence composed only by gamepad buttons.

Defining the ideal maximum acceptable error rates is not easy. With this in mind, we made it possible to also configure these values in the XML grammar.

The joystick phase of the training session can be used to calculate the maximum amplitude achieved by the user. This value can then be used to parameterize the maximum speed value. Imagining a user who can only push the joystick to $50 \%$ of its maximum amplitude, the speed can be calculated by multiplying the axis value by two. This feature was not implemented. However, all the background preparation to implement it was set for future work.

The speech component of the training session was used to define the recognition trust level for each of the voice commands. The trust level is a percentage value retrieved by the speech recognition engine. This value is used to set the minimum recognition level for the recognition module.

Finally, the head movement phase of the training session has a similar purpose to the joystick's phase. Additionally, the maximum amplitude for each direction can be used to determine the range that will trigger each one of the leaning inputs of the head gestures recognition.

\section{EXPERIMENTS AND RESULTS}

The main objective of the experimental work was to make a preliminary study of the tasks that can be implemented and the responses of the individuals in order of get information for the user profiling. The experiments involved 33 voluntaries, with a mean age of 24, a standard deviation of 4.2 and without any movements' restrictions.

The first experiment consisted in performing the sequence tasks with several levels of difficulty. In the first sequence the users needed to push the gamepad buttons GP1 - GP2 (easy level of difficulty); the second sequence was GP3 - GP8 (easy level of difficulty); the third sequence was GP5 - GP8 - GP9 (medium level of difficulty) and the last sequence was GP6 - GP1 - GP7 - GP4 - GP2 (hard level of difficulty). For the experiments with voice commands the individuals had to pronounce the sentences: "Go forward"; "Go back"; "Turn right"; "Turn left"; "Right spin"; Left Spin" and "Stop" to get the information about the recognition trust level for each voice command.

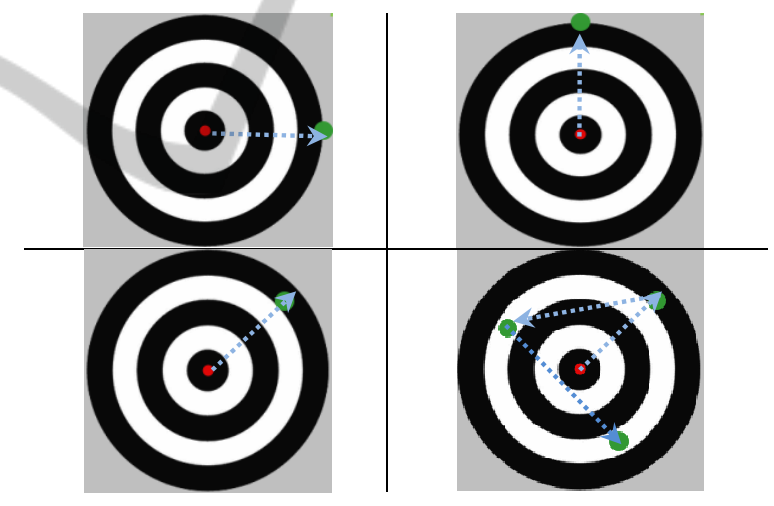

Figure 6: User profiler joystick tests.

The last two experiments involved the precision of the gamepad's joystick and the head movements. The voluntaries should move the small dot into the bigger one with the gamepad's joystick and with the wiimote controller. Figure 6 shows some of the tasks that were asked. The positions were moving right; up; down; northeast; north-west; southeast and a sequence northeast - northwest - southeast without going back to the initial position in the center of the target.

In general, the achieved results show the good performance of the individuals using gamepad and voice commands. The behaviour with head movements reflects more asymmetry and heterogeneous results, since several moderate and severe outliers exist in the time results. The time 
consumed to perform the sequences confirmed the complexity of the tasks as can be seen in Figure 7. In terms of average time between buttons (Figure 8) it is interesting to notice the results for the last sequence. Although it is more complex and longer it has a positive asymmetry distribution. This probably reveals that training may improve the user's performance.

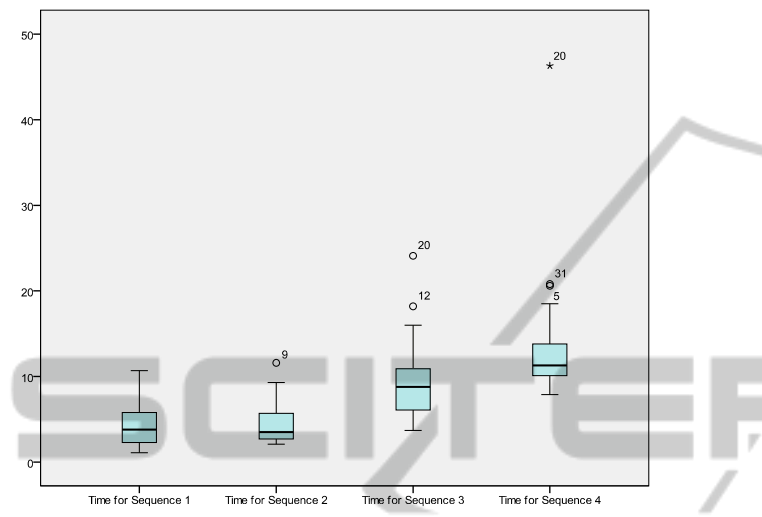

Figure 7: Time to perform the sequences.

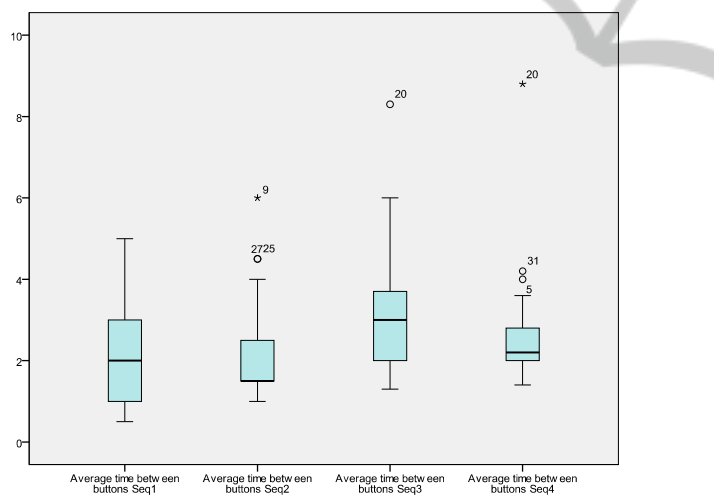

Figure 8: Average time between gamepad buttons.

In terms of errors, the third sequence presents a higher result with at least one fail. The last sequence presented a case where 12 errors were committed.

Table 1: Contingency table with the errors of sequences.

\begin{tabular}{ccccccccc}
\hline & \multicolumn{10}{c}{ Number of Errors } \\
Seq & $\mathbf{0}$ & $\mathbf{1}$ & $\mathbf{2}$ & $\mathbf{3}$ & $\mathbf{4}$ & $\mathbf{5}$ & $\mathbf{6}$ & $\mathbf{1 2}$ \\
\hline 1 & 30 & 1 & 2 & 0 & 0 & 0 & 0 & 0 \\
2 & 31 & 2 & 0 & 0 & 0 & 0 & 0 & 0 \\
3 & 20 & 7 & 3 & 1 & 1 & 0 & 1 & 0 \\
4 & 27 & 1 & 1 & 1 & 0 & 2 & 0 & 1 \\
\hline
\end{tabular}

Table 2 presents several descriptive statistics, such as central tendency (mean, median) and dispersion (standard deviation, minimum and maximum), for the trust level of speech recognition.
Table 2: Descriptive Statistics for the trust level of speech recognition.

\begin{tabular}{cccccc}
\hline Sentence & Mean & Median & S. Dev & Min & Max \\
\hline "Go Forward" & $\mathbf{9 5 . 3 6}$ & $\mathbf{9 5 . 5 0}$ & 0.51 & 93.9 & 95.9 \\
"Go Back" & 94.37 & 95.00 & 2.44 & 82.2 & 95.9 \\
"Turn Right" & 95.31 & 95.40 & $\mathbf{0 . 4 2}$ & 94.4 & 95.9 \\
"Turn Left" & 94.76 & 95.20 & 1.42 & 88.4 & 95.8 \\
"Left Spin" & 93.69 & 94.90 & 2.88 & 83.1 & 95.8 \\
"Right Spin" & 94.82 & 95.00 & 1.25 & 89.7 & 97.2 \\
"Stop" & $\mathbf{9 2 . 6 7}$ & 94.30 & $\mathbf{3 . 8 5}$ & 82.2 & 95.8 \\
Total Sentences & 94.43 & 94.99 & 1.08 & 92.24 & 95.93 \\
\hline
\end{tabular}

The speech recognition has very good results. In fact, the minimum of minimums was 82.2 for the sentences "Go Back" and "Stop". The expression "Go Forward" has the highest mean and median. The sentence "Stop" is more heterogeneous since it has the higher standard deviation (3.85).

The paired samples $t$ test was applied with a significance level of 0.05 to compare the means of time using joystick and head movements. It was established the null hypothesis: the means of time to perform the target tasks with joystick and head movements were equal. The alternative hypothesis is: the means of time to perform the target tasks with joystick and head movements were not different. The achieved power was of 0.80 with an effect size of 0.5 . Table 3 contains the $p$ values of the paired sample $t$ tests and the $95 \%$ confidence interval of the difference. Observing the results for the positions Down and Northwest, it is valid to claim there are statistical evidences to affirm that the mean of time with joystick and head movements is different. This reveals the different performance by using in the same experience the joystick and the head movements.

Clustering analysis is a technique that can be used to obtain the information about similar groups. In the future, this can be used to extract characteristics for classification and users' profiling.

The results obtained by hierarchical clustering, using the nearest neighbour method and squared Euclidean distance, show the similar performance of subjects except one individual. In this case, using the

Table 3: Confidence intervals of the difference and $p$ values.

\begin{tabular}{|c|c|c|c|}
\hline \multirow[b]{2}{*}{ Move the red dot to: } & \multicolumn{2}{|c|}{$\begin{array}{l}95 \% \text { Confidence Interval of } \\
\text { the difference }\end{array}$} & \multirow[b]{2}{*}{$\begin{array}{c}\mathbf{P} \\
\text { value }\end{array}$} \\
\hline & Lower & Upper & \\
\hline Right & -2.29 & 0.67 & 0.273 \\
\hline Up & -1.38 & 0.08 & 0.080 \\
\hline Down & -9.67 & -1.87 & $0.005^{*}$ \\
\hline Northeast & -2.89 & 0.66 & 0.211 \\
\hline Northwest & -2.74 & -0.17 & $0.028^{*}$ \\
\hline Southeast & -6.26 & 1.00 & 0.150 \\
\hline $\begin{array}{c}\text { Northeast - Northwest - } \\
\text { Southeast }\end{array}$ & -5.32 & 0.37 & 0.085 \\
\hline
\end{tabular}


R-square criteria, the number of necessary clusters to achieve $80 \%$ of the total variability retain by the clusters is 12 . Since the sample of volunteers was from the same population, this kind of conclusions are very natural. So the next step will consist in obtain information about handicapped people. In fact, if the clusters of subjects could be defined then it should be interesting to work with supervised classification in which the best command mode would be the class.
FCT - Portuguese Science and Technology Foundation for the INTELLWHEELS project funding (RIPD/ADA/109636/2009), for the $\mathrm{PhD}$ Scholarship FCT/SFRH/BD/44541/2008, LIACC Laboratório de Inteligência Artificial e de Computadores, DETI/UA - Dep. Electrónica, Telecomunicações e Informática and ESTSP/IPP Escola Superior de Tecnologia da Saúde Porto IPP.

\section{CONCLUSIONS AND FUTURE WORK}

Although many Intelligent Wheelchair prototypes are being developed in several research projects around the world, the adaptation of user interfaces to each specific patient is an often neglected research topic. Typically, the interfaces are very rigid and adapted to a single user or user group. Intellwheels project is aiming at developing a new concept of Intelligent Wheelchair controlled using high-level commands processed by a multimodal interface. However, in order to fully control the wheelchair, users must have a wheelchair interface adapted to their characteristics. In order to collect the characteristics of individuals it is important to have variables that can produce a user profile. The first stage must be a statistical analysis to extract knowledge of user and the surrounding. The second stage must be a supervised classification to use Machine Learning algorithms in order to construct a model for automatic classification of new cases.

This paper mainly refers to the proposal of a set of tasks for extracting the required information for generating user profiles. A preliminary study has been done with several voluntaries, enabling to test the proposed methodology before going to the field and acquiring information with disabled individuals. In fact, this will be the next step for future work. The test set presented in this paper will be tested by a group of disabled individuals, and the results of both experiments will be compared to check if the performances of both populations are similar. Also, in order to collect feedback regarding the system usability, disabled users will be invited to drive the wheelchair in a number of real and simulated scenarios.

\section{ACKNOWLEDGEMENTS}

The authors would like to acknowledge to

\section{REFERENCES}

Adachi, Y., Kuno, Y., Shimada, N., Shirai, N., 1998. Intelligent wheelchair using visual information on human faces. In International Conference in Intelligent Robots and Systems, vol. 1, pp. 354-359.

Bell, D. A., Borenstein, J., Levine, S. P., Koren, Y.; Jaros, J., 1994. An assistive navigation system for wheelchairs based upon mobile robot obstacle avoidance. In IEEE Conf. on Robotics and Automation, pp. 2018-2022.

Borgerding, B., Ivlev, O., Martens, C., Ruchel, N., Gräser, A., 1999. FRIEND: Functional robot arm with user friendly interface for disabled people. In 5th European Conf. for the Ad-vancement of Assistive Technology.

Braga, R., Petry, M., Moreira, A.P., Reis, L.P., 2009. Concept and Design of the Intellwheels Platform for Developing Intelligent Wheelchairs. In LNEE/ Informatics in Control, Automation and Robotics, vol. 37, pp. 191-203.

Cypher, A., Halbert, D. C., 1994. Watch what I do: programming by demonstration. A. Cypher and D. C. Halbert, Eds. Massachusetts, USA: Library of Congress.

Faria, P.M., Braga, R., Valgôde, E., Reis, L.P., 2007. Interface framework to drive an intelligent wheelchair using facial expressions. In IEEE International Symposium on Industrial Electronics, Vigo, pp. 17911796.

Gao, C., Hoffman, I., Miller, T., Panzarella, T., Spletzer, J., 2008. Performance Characterization of LIDAR Based Localization for Docking a Smart Wheelchair System. In Internationsl Conference on Intelligent Robots and Systems, San Diego.

Hamagami, T., Hirata, H., 2004. Development of Intelligent Wheelchair acquiring autonomous, cooperative and collaborative behaviour. In IEEE International Conference on Systems Man and Cybernetics, vol. 4, pp. 3525-3530.

Horn, O., Kreutner, M., 2009. Smart wheelchair perception using odometry, ultrasound sensors and camera. Robotica, vol. 27, no. 2, pp. 303-310, March.

Hoyer, H., Hölper, R., 1993. Open control architecture for an intelligent omnidirectional wheelchair. In Proc.1st TIDE Congress, Brussels, pp. 93-97.

Jia, P., Hu, H., Lu, T., Yuan, K., 2007. Head Gesture Recognition for Hands-free Control of an Intelligent 
Wheelchair. Journal of Industrial Robot, vol. 34, no. 1, pp. 60-68.

Jia, P., Hu, H., Lu, T., Yuan, K., 2007. Head Gesture Recognition for Hands-free Control of an Intelligent Wheelchair. Journal of Industrial Robot, vol. 34, no. 1.

Lakany, H., 2005. Steering a wheelchair by thought. IEE Digest, vol. 2005, no. 11059, pp. 199-202, The IEE International Workshop on Intelligent Environments.

Langley, P., 1997. Machine learning for adaptive user interfaces. In Proceedings of the 21st Ger-man Annual Conference on Artificial Intelligence, Freiburg, p. 5362.

Langley, P., 1999. User modeling in adaptive interface. In Proceedings of the seventh international conference on User modeling, Banff, pp. 357-370.

Levine, S. P., Bell, D. A., Jaros, L. A., Simpson, R. C., Koren, Y., 1999. The NavChair assistive wheelchair navigation system. In IEEE Transactions on Rehabilitation Engineering, vol. 7, pp. 443-451.

Madarasz, R. L., Heiny, L. C., Cromp, R. F., Mazur, N. M., 1986. The design of an autonomous vehicle for the disabled. IEEE Journal of Robotics and Automation, vol. 2, no. 3, pp. 117-126, September.

Maes, P., 1996. Intelligent Software: Programs That Can Act Independently Will Ease the Burdens that Computers Put on People. IEEE Expert Systems, vol. 11, no. 6, pp. 62-63, February.

Miller D., Slack, M., 1995. Design and testing of a lowcost robotic wheelchair. In Autonomous Robots, vol. 2, pp. 77-88.

Miller, D.P., 1998. Assistive Robotics: An Overview. In Assistive Technology and AI, pp. 126-136.

Ng, P. C., De Silva, L. C., 2001. Head gestures recognition. In Proceedings International Conference on Image Processing, pp. 266-269.

Norcio, A. F., Stanley, J., 1989. Adaptive HumanComputer Interfaces: A Literature Survey and Perspective. IEEE Transactions on Systems, Man and Cybernetics, vol. 19, no. 2, pp. 399-408, March.

Pruski, A., Ennaji, M., Morere, Y., 2002. VAHM: A user adapted intelligent wheelchair. In Proceedings of the 2002 IEEE International Conference on Control Applications, Glasgow, p. 784789.

Rebsamen B., Rebsamen, B., Burdet, E., Guan, C., Haihong Zhang, Chee Leong Teo, Qiang Zeng, Ang, M., Laugier, C., 2006. A Brain-Controlled Wheelchair Based on P300 and Path Guidance. In IEEE/RASEMBS International Conference, vol. 20, pp. 11011106.

Reis, L. P., Braga, R., Sousa, M., Moreira, A. P., 2010. Intellwheels MMI: A Flexible Interface for an Intelligent Wheelchair. RoboCup 2009: Robot Soccer World Cup XIII, Springer Ber-lin/Heidelberg, LNCS, vol. 5949, Graz, pp. 296-307.

Ross, E., 2000. Intelligent User Interfaces: Survey and Research Directions. University of Bristol, Bristol, Technical Report: CSTR-00-004.

Satoh Y., Sakaue, K., 2007. An Omnidirectional Stereo Vision-Based Smart Wheelchair. EURASIP Journal on Image and Video, p. 11.
Simpson, R., 1998. NavChair: An Assistive Wheelchair Navigation System with Automatic Adaptation. In Assistive Technology and Artificial Intelligence. Berlin: Springer-Verlag Berlin Heidelberg, p. 235.

Simpson, R. C., 2005. Smart wheelchairs: A literature review. Journal of Rehabilitation Research and Development, vol. 42 (4), pp. 423-436.

Volosyak, I., Ivlev, O., Graser, A., 2005. Rehabilitation robot FRIEND II - the general concept and current implementation. In ICORR 2005 - 9th International Conference on Rehabilitation Robotics, Chicago, pp. 540-544.

Wellman, P., Krovi, V., Kumar, V., 1994. An adaptive mobility system for the disabled. In Proc. IEEE Int. Conf. on Robotics and Automation.

Wooldridge, M., 2002. An Introduction to Multi-Agent Systems. John Wiley \& Sons.

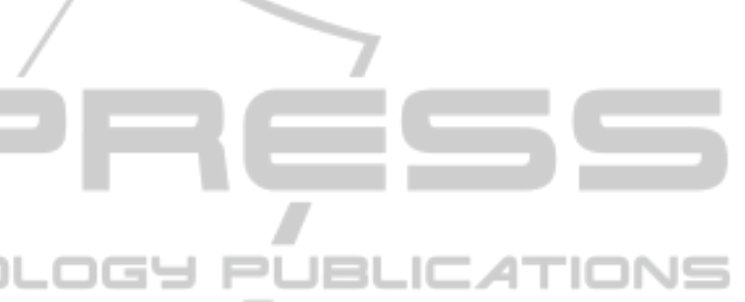

